\title{
Does Transdermal Testosterone Increase the Risk of Developing Breast Cancer? A Systematic Review
}

\author{
RITIKA GERA, SALIM TAYEH, HIBA EL-HAGE CHEHADE and KEFAH MOKBEL
}

London Breast Institute, London, U.K.

\begin{abstract}
Background/Aim: Hypoactive sexual desire disorder (HSDD) is hypothesised to manifest in postmenopausal women at onset of menopause due to decreased oestrogen levels. Transdermal testosterone is a potential treatment option. This systematic review explores the relationship between the incidence of breast cancer and transdermal testosterone use. Materials and Methods: Searches were conducted on the PubMed and Ovid databases. In Ovid, the advanced search function was used: 'transdermal testosterone not male'. In PubMed, the following search terms were used: 'transdermal, testosterone, menopausal, women, breast cancer, women'. Abstracts that fitted our initial criteria were further investigated. Results: A total of 25 publications from PubMed and 192 publications from Ovid were initially assessed. Three randomised control trials were judged to have sufficiently met our inclusion criteria. However, these trials were too heterogeneous for a meta-analysis. A systematic review was deemed the most appropriate analysis of the data available. Conclusion: The publications examined in this systematic review suggest that the use of transdermal testosterone to treat $H S D D$ in postmenopausal women does not increase breast cancer incidence. However, further research in the form of adequately powered randomised controlled trials with breast cancer incidence being the primary end point is required in order to confirm this.
\end{abstract}

Hypoactive sexual desire disorder (HSDD) is likely to be the most common sexual affliction affecting midlife women (1);

This article is freely accessible online.

Correspondence to: Kefah Mokbel, London Breast Institute, 42 52 Nottingham Place, Marylebone, London, W1U 5NY, U.K. Tel: +44 2079082040, Fax: +44 2079082275, e-mail: kefahmokbel@ hotmail.com

Key Words: Hypoactive sexual desire disorder, transdermal testosterone, breast cancer, postmenopausal, review. it manifests by causing "marked distress or interpersonal difficulty" in the absence of desire for sexual activities and sexual fantasies (2). Transdermal testosterone (TT) patches or gels have been suggested as an effective treatment for HSDD in postmenopausal women (3-5).

Intrinsa is a TT patch indicated for use in bilaterally oophorectomized and hysterectomised women using concomitant oestrogen therapy and suffering from HSDD. However, a study in 2014 found that only $20.9 \%$ patients included in their cohort were prescribed Intrinsa according to the recommendations of the manufacturer (6). This lends credibility to the notion that the safety and efficacy of transdermal testosterone in treating all artificially and naturally postmenopausal women must be thoroughly assessed to ensure there is no danger in its prescription to treat HSDD.

A randomised controlled trial conducted on 533 postmenopausal women suffering from HSDD concluded that patients using testosterone patches for 24 weeks had significantly improved sexual desire $(p<0.001)$ and significantly decreased personal distress (7). There are many publications that support the correlation between the use of TT to treat HSDD and a significant increase in sexual desire (8-11).

However, the use of TT treatment for HSDD raises a key safety concern; theoretically, there is an increased risk of breast cancer development in postmenopausal patients due to raised concentrations of oestradiol. There is also the concern that women who have triple-negative breast cancer are at high risk of worsening their disease by using transdermal testosterone to treat their HSDD.

A randomised controlled trial investigated whether a 5 or $10 \mathrm{mg}$ dose of transdermal testosterone cream (TTC) per day was required to effectively treat HSDD (12). Seven healthy postmenopausal women were randomly allocated to receive either 5 or $10 \mathrm{mg}$ of TTC per day. It was found that the $5 \mathrm{mg}$ dose was sufficient to restore serum total testosterone and free testosterone levels to within and above the reference range for premenopausal women. Moreover, in the post-dose sampling period, neither dose resulted in any meaningful variation in multiple influential sex hormones: dihydrotestosterone, 
estradiol, estrone, and sex hormone-binding globulin. This suggests that the dose of TTC required to treat HSDD could potentially be lessened without impacting its effect on HSDD to decrease the risk of adverse events in susceptible patients.

There is some compelling laboratory evidence present in the literature suggesting that transdermal testosterone may have a protective role against the development of breast cancer. Questioning the safety of combined estrogen/ progesterone therapy, a randomised double blinded placebo trial was conducted to investigate whether the addition of a $300 \mu \mathrm{g}$ testosterone patch to combined estradiol $2 \mathrm{mg} /$ norethisterone acetate $1 \mathrm{mg}$ would affect breast cell proliferation (13). A total of 88 post-menopausal female patients were used. In the placebo group, the increase in total breast cell proliferation was observed as being more than fivefold $(p<0.001)$. In direct contrast, with the addition of the testosterone patch, no significant increase was detected. The findings were apparent in both epithelial and stromal cells.

A randomised trial investigated the use of intravaginal testosterone cream versus estradiol vaginal ring for women receiving aromatase inhibitors for early-stage breast cancer to see if they could improve vaginal dryness or decreased libido (14). Despite a very small sample of only 69 patients completing treatment, the study compellingly illustrated that estradiol elevation was not present in any patient using the vaginal ring and only present in a small proportion of patients using the testosterone cream.

A consensus is yet to be reached on the safety of transdermal testosterone use for postmenopausal women and the nature of its relationship with breast cancer. Our systematic review aims to tackle this uncertainty.

\section{Materials and Methods}

Articles deemed relevant were identified using electronic database searches on PubMed and Ovid. In both searches, no upper limit was specified. In Ovid, the following search terms were used: transdermal testosterone not male. Multiple databases were searched: AMED, Embase (1974-July 13, 2018), Ovid MEDLINE(R) and Epub Ahead of Print, In-Process \& Other Non-Indexed Citations, Daily and Versions ${ }^{\circledR}$ (1946-July 13, 2018). There were 280 results generated in total but 192 results were analysed post deduplication. In PubMed, the search terms were: transdermal, testosterone, menopausal, women, and breast cancer. There were 25 search results. Two assessors undertook independent systematic searches and evaluated the abstracts to select the studies for review. The primary aim of these searches was to locate clinical trials that examined the use of transdermal testosterone in postmenopausal women and recorded breast cancer incidence as an endpoint in patients using transdermal testosterone or placebo. The citations of other reviews and meta-analyses were also assessed to determine if there were any randomised controlled trials that fitted our inclusion criteria.

Inclusion and exclusion criteria. The trials included must have been randomised clinical trials or retrospective case control studies. They should have examined postmenopausal (natural or surgically induced) female patients. In a randomised control trial investigating the risk associated with transdermal testosterone use, an endpoint investigated was the incidence of breast cancer; this did not have to be the primary endpoint. In a retrospective case control study investigating the incidence of breast cancer, the key risk factor investigated must have been the use of transdermal testosterone. If the study performed on an interview basis then at the time of interview all cases and controls must have been alive and have conducted the interview in person themselves. Investigation into postmenopausal women must have been mentioned in the abstract.

The study was excluded if any of the following criteria were met: the full text cannot be found, available data regarding controls has not been published, and there were patients who had been given a diagnosis of any other cancer apart from that of the breast OR if there were patients for whom this was not the first diagnosis of breast cancer in a retrospective study. Any data regarding male or pre-menopausal patients were excluded.

\section{Results}

Three randomised controlled trials met our inclusion criteria and evaded our exclusion criteria (15-17). Our original aim was to conduct a meta-analysis assessing whether there is a significant association between the incidence of breast cancer and the use of transdermal testosterone to treat HSDD in postmenopausal patients. However, the data from these three studies was incomparable due to their significant heterogeneity.

Nachtigall et al., included women prescribed with oestrogen whereas Davis et al., did not and Panay et al., excluded the oestrogen criteria to increase the size of their sample. Nachtigall et al., and Davis et al., included women between the ages of 20 and 70 whereas Panay et al., included women between the ages of 40 and 70 .

The purpose of a meta-analysis should be to aggregate a treatment effect (a comparison between treated and non-treated). After screening the papers for these data, such information can clearly be extracted from Davis et al., and Panay et al., also provided enough suitable data because the researchers reported on the incidence of breast cancer. However, after twenty-four weeks in the Nachtigall et al., study, women who decided to continue into the open-label experiment were treated with transdermal testosterone; there was no method by which the patients could be apportioned between treated and non-treated groups.

We were able to conduct combined analysis (chi squared test) using the data from these three studies (15-17). There were no placebo users from the Nachtigall et al., study, hence, a sample size equal to the treatment group was used to calculate the expected number of breast cancer cases. The chi squared statistic was 2.0848 , with a p-value of 0.148776 , hence this result is not statistically significant at $p<0.05$ (Table I).

\section{Discussion}

To examine the tolerability and safety profile of testosterone patch therapy in conjunction with oral or transdermal oestrogen, a clinical trial was conducted on 641 surgically 
Table I. Chi Squared calculation to determine the relationship between breast cancer cases and TT use.

\begin{tabular}{lccc}
\hline & TT patch users & Non-TT patch users & Marginal row totals \\
\hline $\begin{array}{l}\text { Observed cases of breast cancer in TT patch users, } \\
\text { expected cases of breast cancer in placebo users }\end{array}$ & $9(6.52)[0.95]$ & $3(5.48)[1.13]$ & 12 \\
Number of patients & $1,058(1,060.48)[0.01]$ & 895 (892.52) [0.01] & 1953 \\
Marginal column totals & 1,067 & 898 & 1,965 (Grand Total) \\
\hline
\end{tabular}

menopausal women to completion for up to four years (15). All patients were receiving TT therapy in this open-label trial after six months. This study concluded that there was no significant increase in the occurrence of major adverse effects. Milder side effects, such as reactions to the application site and unwanted hair growth, were more common and unlikely to result in the termination of therapy. Three cases of invasive breast cancer were observed in patients on TT patch therapy, but these levels were consistent with age-appropriate expected rates. Furthermore, there were four cases of ductal carcinoma in situ observed amongst TT patch therapy users. However, one of these cases was diagnosed one year after the patient discontinued her use of TT patch therapy. This trial was the continuation of two earlier trials $(7,10)$; the switch to open-label occurred after patients spent six months respectively on TT $(7,10)$.

This trial did not specifically focus on breast cancer as a key risk factor. It was examined as one of multiple adverse effects. The main demographic examined in this trial was women with surgical menopause post-oophorectomy, hence further evidence is required to conclude whether these results can be replicated in naturally post-menopausal women. Of the seven patients diagnosed with breast cancer, six were initially randomised to placebo in the first six months $(7,10)$ before continuing the open-label treatment. There was also no breast examination or mammogram conducted on these six patients prior to initiation of TT patch therapy because this was not part of the protocol. Therefore, there is no way to definitively conclude whether breast cancer in these six cases was caused by TT patch therapy or whether they were pre-existing issues which were identified during TT patch therapy. Ninenteen percent of patients chose to undergo voluntary withdrawal from the treatment arm. The investigators explained this phenomenon by suggesting it was because each successive year of investigation following on from the initial 6 or 12-month study design required additional consent.

To examine the effect of TT therapy versus placebo therapy on post-menopausal women with HSDD, a doubleblind placebo-controlled 52-week trial using 464 postmenopausal women was conducted to completion over the course of 52 weeks (16). Four cases of breast cancer were diagnosed in the group receiving testosterone as opposed to no cases in the placebo group. Of the women who developed breast cancer, one retrospectively had pre-randomisation symptoms (a bloody discharge from the nipple) and another was diagnosed within the initial four months of study initiation. The patient with bloody nipple discharge was excluded from our statistical analysis because she had preexisting symptoms that suggest she had breast cancer prior to TT therapy initiation. One patient who undertook treatment with $300 \mu \mathrm{g}$ of testosterone/day for 104 weeks (the study offered treatment extension subject to follow-up) was diagnosed with infiltrating ductal breast carcinoma 3 months post study cessation by mammography; the 52-week mammography was, however, reported as normal. Patients from the study extension could not be included in our statistical analysis because the authors of the study did not provide information regarding patient distribution between the treatment arm and the placebo arm.

The primary endpoint examined in this study (16) was a change from baseline sexual satisfaction in four-week intervals up to 24 weeks. The increase in satisfaction was significantly higher in women receiving $300 \mu \mathrm{g}$ of testosterone compared to women receiving $150 \mu \mathrm{g}$, but both doses resulted in significantly higher satisfaction levels when compared to women who were part of the placebo group.

Two separate groups of women were used in this study (16): women with surgically-induced menopause between the ages of 20 and 70 and women between the ages of 40 and 70 who had been naturally post-menopausal for at least two years. Only $57 \%$ of patients opted to complete the full 52 weeks so only 464 women were followed to completion. However, the investigators rationalised this by suggesting that the effect of patient withdrawal can be offset by the relatively large sample size. Screening participants under the age of 40 for normal mammograms was not a requirement for the study. Patients were excluded if they had used any systemic oestrogen/oestrogen-progestin therapy over the three-month period prior to study commencement. However, it is possible that a longer period of abstinence from oestrogen related therapy should have been required to minimise its effect on breast cancer development. One of the patients who developed breast cancer reported that her sister had also suffered from 
the disease, hence the patient may have been at an increased risk independent of the transdermal testosterone therapy.

The ADORE study (17) examined 272 naturally menopausal women who were randomised to receive TT patch or placebo twice a week for 6 months. This multicentred and double-blind study was conducted on patients who were, for the most part, not on hormone therapy. Once again, efficacy measures were conducted at the 4-week marks by looking at sexual desire through a Sexual Activity Log. $76 \%$ of patients completed 24 weeks on the study and the overall mean exposure to TT patch was 147 days. Within this study, there were no myocardial infarctions, deaths or breast cancers reported. This may have been due to the relatively small cohort and the lack of any follow up period post study termination.

Mammographic density is a risk factor closely correlated with breast cancer development. A recent study has suggested that an increased density may represent an intermediate stage in breast cancer development for some biological pathways (18). A clinical trial which examined the impact of the use of TT therapy on the mammographic density of postmenopausal women (19) therefore provides a valuable indication of breast cancer risk for this cohort of patients. This 52-week study used 279 postmenopausal women who were not using any systemic oestrogen/oestrogen-progestin therapy up to three months prior to trial initiation. Mammographic examinations were conducted at screening (week 4) and at study termination (week 52) or exit (assuming withdrawal did not occur prior to week 24). For cumulative dense or non-dense areas, there were no statistically different results for the group treated with 150 or $300 \mu \mathrm{g} /$ day TT patch compared to the placebo group. While this study did not use the diagnosis of breast cancer or lack thereof as a definitive endpoint, it clearly indicates that there is little statistically significant increased risk between transdermal testosterone application and mammographic density.

Aromatase inhibitors are commonly used by women with hormone receptor sensitive breast cancer (20). However, vaginal dryness and, in some cases, vaginal atrophy may present as a side effect of the aromatase inhibitors. A total of 21 postmenopausal women with breast cancer who were being treated with aromatase inhibitors were given $300 \mu \mathrm{g}$ or $150 \mu \mathrm{g}$ of TT cream. Estradiol remained inhibited $(<8 \mathrm{pg} / \mathrm{ml})$ post treatment and mean symptom total scores significantly improved $(p<0.001)$. This treatment was not aimed at women with HSDD and it was used in women who had already developed breast cancer, but it indicates that transdermal testosterone cream can safely and effectively be used in women with hormone sensitive breast cancer in combination with an aromatase inhibitor. This finding is supported in the wider literature (21). However, due to the small sample size and short duration of treatment, further experimentation is required to determine the long-term effects of TT therapy on women with estrogen receptor- positive breast cancer. Caution should be also exercised when considering a use of TT in patients with triple-negative breast cancer that is positive for androgen receptor expression.

A retrospective trial conducted by Davis et al. (22) concludes that the use of testosterone was not significantly associated with increased breast cancer risk. From a sample of 631 women taking testosterone therapy, twelve cases of invasive breast cancer were found during follow up, and only one of these patients was using transdermal testosterone therapy alone. Another patient was using the testosterone implant and testosterone cream simultaneously.

Prospective trials into the safety of Libigel (a testosterone gel) from the perspective of cardiovascular and oncological risk are ongoing (23). Further prospective trials are required to come to a significant conclusion on the safety profile of testosterone gel.

A recent qualitative systematic review examining transdermal testosterone usage in female HSDD (24) drew largely similar conclusions to our systematic review. Of the five studies examined in the review conducted by Ganesan et al., only three use breast cancer as an endpoint and we have included and analysed those three studies in this systematic review (15-17). The earlier systematic review (24) did not focus specifically on breast cancer risk and instead broadly examined the efficacy of transdermal testosterone in producing an increase in sexual desire and activity. Ours is the only systematic review with a key focus of examining breast cancer risk in relation to transdermal testosterone application for postmenopausal women.

Many systematic reviews were examined to determine pre-existing notions regarding the relationship between TT therapy and breast cancer incidence in treating hypoactive sexual desire disorder in postmenopausal women (25-33). There is clearly a need to further investigate any potential safety risks related with the use of transdermal testosterone, particularly by using randomised prospective trials.

Recommendations for future trials investigating the relationship between transdermal testosterone and breast cancer in postmenopausal women. A double-blind prospective trial with a large cohort of postmenopausal women who do not have any previous history of cancer should be conducted. Participants should be excluded if they have used any systemic oestrogen/oestrogen-progestin treatment in the past three months or if they have any testosterone implants. They should then be stratified to filter out the confounding effect of risk factors for breast cancer such as BMI and ethnicity and subsequently be randomised to receive either the placebo or varying concentrations of transdermal testosterone treatment. Participants should be followed up for at least a year and checked for the development of breast cancer within the years following study termination. 
There must be a standardised method of testing for the development of breast cancer throughout the trial Mammographic testing prior to study initiation and upon study termination must happen. If women display symptoms of breast cancer prior to study initiation $(34,35)$ they must be excluded. This can be determined from a standardised survey conducted prior to study initiation. A PAP smear must also be conducted prior to study initiation to determine the risk of developing cervical cancer throughout the course of the investigation. If the PAP smear or the initial mammography shows clinically suspicious results, then the participant must be excluded from the study and referred for specialist consultation.

Participants should be randomised to receive varying concentrations of transdermal testosterone or placebo. Participants should be asked to keep a sexual desire and activity journal to document any increase in their arousal levels due to the transdermal testosterone treatment. They must have a steady sexual partner who is physically present for at least $50 \%$ of the month. An in-person interview should be conducted monthly to chart progress and record the results from the journal. This would be preferred to an impersonal survey because it increases the chances that patients will be more truthful in reporting the successes/ failures they have experienced with the treatment; such confidential information is better elicited in person. Once the investigation has concluded, a full report containing data regarding placebo and transdermal testosterone treated patients must be published.

\section{Conclusion}

There is no evidence that transdermal testosterone significantly increases the risk of breast cancer development in postmenopausal women. However, the studies included in our systematic review have significant limitations. These encompass: the incidence of breast cancer in postmenopausal women not being the primary endpoint or focus of the investigation, limited if any follow up post termination of transdermal testosterone therapy, and a lack of standardised breast screening protocols. There are no reliable data available for high-risk patients such as post-menopausal women who are at an increased risk of developing hormone sensitive breast cancer.

If a postmenopausal breast cancer patient is receiving adjuvant endocrine therapy then transdermal testosterone is unlikely to have a negative impact. However, women who have hormone sensitive breast cancer should be wary of using transdermal testosterone. Monitoring serum estradiol and testosterone levels to avoid high levels should help to ensure the safety of the treatment.

There is currently not enough evidence to definitively state that there is no increased risk of breast cancer associated with the prescription of transdermal testosterone to postmenopausal women. However, the evidence we have examined suggests a positive safety profile for this treatment. Further research is required to confirm our tentative conclusion.

\section{References}

1 Worsley R, Bell RJ, Gartoulla P and Davis SR: Prevalence and predictors of low sexual desire, sexually related personal distress, and hypoactive sexual desire dysfunction in a community-based sample of midlife women. J Sex Med 14(5): 675-686, 2017.

2 Warnock JJ: Female hypoactive sexual desire disorder: epidemiology, diagnosis and treatment. CNS Drugs 16(11): 745753, 2002.

3 Nappi R, Wawra K and Schmitt S: Hypoactive sexual desire disorder in postmenopausal women. Gynaecological Endocrinology 22(6): 318-323, 2006.

4 Ganesan K, Habboush Y and Sultan S: transdermal testosterone in female hypoactive sexual desire disorder: a rapid qualitative systematic review using grading of recommendations assessment, development and evaluation. Cureus 10(3): e2401, 2018.

5 Nappi R, Martini E, Terreno E, Albani F, Santamaria V, Tonani S, Chiovato L and Polatti F: Management of hypoactive sexual desire disorder in women: current and emerging therapies. Int $\mathbf{J}$ Womens Health 2: 167-175, 2010.

6 Osborne V, Layton D and Shakir SA: Implications of off-label use: An example from the final results of an observational cohort study on Intrinsa ${ }^{\circledR}$ (testosterone patch). Post Reprod Health 20(2): 48-54, 2014.

7 Buster JE, Kingsberg S, Aguirre O, Brown C, Breaux J, Buch A, Rodenberg C, Wekselman $\mathrm{K}$ and Casson P: testosterone patch for low sexual desire in surgically menopausal women: A randomized trial. ObstetGynaecol 105(5 Pt 1): 944-952, 2005.

8 Panay N, Al-Azzawi F, Bouchard C, Davis SR, Eden J, Lodhi I, Rees M, Rodenberg CA, Rymer J, Schwenkhagen A and Sturdee DW: Testosterone treatment of HSDD in naturally menopausal women: the ADORE study. Climacteric 13(2): 121-131, 2010.

9 Shifren JL, Davis SR, Moreau M, Waldbaum A, Bouchard C, DeRogatis L, Derzko C, Bearnson P, Kakos N, O'Neill S, Levine $\mathrm{S}$, Wekselman $\mathrm{K}$, Buch $\mathrm{A}$, Rodenberg $\mathrm{C}$ and Kroll R: Testosterone patch for the treatment of hypoactive sexual desire disorder in naturally menopausal women: results from the INTIMATE NM1 Study. Menopause 13(5): 770-779, 2006.

10 Simon J, Braunstein G, Nachtigall L, Utian W, Katz M, Miller S, Waldbaum A, Bouchard C, Derzko C, Buch A, Rodenberg C, Lucas J, and Davis S: Testosterone patch increases sexual activity and desire in surgically menopausal women with hypoactive sexual desire disorder. J Clin Endocrinol Metabol 90(9): 5226-5233, 2005.

11 Kingsberg S: Testosterone Treatment for Hypoactive Sexual Desire Disorder in Postmenopausal Women. Sexual Health and the Menopause 4(3): 227-234, 2007.

12 Fooladi E, Reuter SE, Bell RJ, Robinson PJ and Davis SR: Pharmacokinetics of a transdermal testosterone cream in healthy postmenopausal women. Menopause 22(1): 44-49, 2015.

13 Hofling M, Hirschberg AL, Skoog L, Tani E, Hägerström T and von Schoultz B: Testosterone inhibits estrogen/progestogeninduced breast cell proliferation in postmenopausal women. Menopause 14(2): 183-190, 2007. 
14 Melisko ME, Goldman ME, Hwang J, De Luca A, Fang S, Esserman LJ, Chien AJ, Park JW and Rugo HS: vaginal testosterone cream vs. estradiol vaginal ring for vaginal dryness or decreased libido in women receiving aromatase inhibitors for early-stage breast cancer: A Randomized Clinical Trial. JAMA Oncol 3(3): 313-319, 2017.

15 Nachtigall L, Casson P, Lucas J, Schofield V, Melson C and Simon JA: Safety and tolerability of testosterone patch therapy for up to 4 years in surgically menopausal women receiving oral or transdermal oestrogen. Gynecol Endocrinol 27(1): 39-58, 2011.

16 Davis SR, Moreau M, Kroll R, Bouchard C, Panay N, Gass M, Braunstein GD, Hirschberg AL, Rodenberg C, Pack S, Koch H, Moufarege A, Studd J and the APHRODITE Study Team: Testosterone for low libido in postmenopausal women not taking estrogen. N Engl J Med 359(19): 2005-2017, 2008.

17 Panay N, Al-Azzawi F, Bouchard C, Davis SR, Eden J, Lodhi I, Rees M, Rodenberg CA, Rymer J, Schwenkhagen A and Sturdee DW: Testosterone treatment of HSDD in naturally menopausal women: the ADORE study. Climacteric 13(2): 121-131, 2010.

18 Rice MS, Bertrand KA, VanderWeele TJ, Rosner BA, Liao X, Adami HO and Tamimi RM: Mammographic density and breast cancer risk: a mediation analysis. Breast Cancer Res 18(1): 94, 2016.

19 Davis SR, Hirschberg AL, Wagner LK, Lodhi I and von Schoultz B: The effect of transdermal testosterone on mammographic density in postmenopausal women not receiving systemic estrogen therapy. J Clin Endocrinol Metab 94(12): 4907-4913, 2009.

20 Fabian CJ: The what, why and how of aromatase inhibitors: hormonal agents for treatment and prevention of breast cancer. Int J Clin Pract 61(12): 2051-2063, 2007.

21 Dahir M and Travers-Gustafson D: Breast cancer, aromatase inhibitor therapy, and sexual functioning: a pilot study of the effects of vaginal testosterone therapy. Sex Med 2(1): 8-15, 2014.

22 Davis S, Wolfe R, Farrugia H, Ferdinand A, Bell R: ORIGINAL RESEARCH-EPIDEMIOLOGY: The Incidence of Invasive Breast Cancer Among Women Prescribed Testosterone for Low Libido. The Journal of Sexual Medicine 6(7): 1850-1856, 2009.

23 White WB, Grady D, Giudice LC, Berry SM, Zborowski J, Snabes MC: A cardiovascular safety study of LibiGel (testosterone gel) in postmenopausal women with elevated cardiovascular risk and hypoactive sexual desire disorder. Am Heart J 163(1): 27-32, 2012.
24 Ganesan K, Habboush Y and Sultan S: Transdermal testosterone in female hypoactive sexual desire disorder: a rapid qualitative systematic review using grading of recommendations assessment, development and evaluation. Cureus 10(3): e2401, 2018.

25 Braunstein GD: Safety of testosterone treatment in postmenopausal women. Fertility and Sterility 88(1): 1-17, 2007.

26 Achilli C, Pundir J, Ramanathan P, Sabatini L, Hamoda H and Panay N: Efficacy and safety of transdermal testosterone in postmenopausal women with hypoactive sexual desire disorder: a systematic review and meta-analysis. Fertil Steril 107(2): 475482, 2017.

27 Kenemans P and van der Mooren MJ: Androgens and breast cancer risk. Gynecol Endocrinol 1: 46-49, 2012.

28 Krapf JM and Simon JA: The role of testosterone in the management of hypoactive sexual desire disorder in postmenopausal women. Maturitas 63(3): 213-219, 2009.

29 Shufelt CL and Braunstein GD: Safety of testosterone use in women. Maturitas 63(1): 63-66, 2009.

30 Kingsberg SA, Simon JA and Goldstein I: The current outlook for testosterone in the management of hypoactive sexual desire disorder in postmenopausal women. J Sex Med 5: 182-193, 2008.

31 Basaria S and Dobs AS: Clinical review: Controversies regarding transdermal androgen therapy in postmenopausal women. J Clin Endocrinol Metab 91(12): 4743-4752, 2006.

32 Somboonporn W: Testosterone therapy for postmenopausal women: efficacy and safety. Semin Reprod Med 24(2): 115-124, 2006.

33 Basaria S and Dobs AS: Safety and adverse effects of androgens: how to counsel patients. Mayo Clin Proc 79: S25-S32, 2004.

34 Shah R, Rosso K and Nathanson SD: Pathogenesis, prevention, diagnosis and treatment of breast cancer. World $\mathrm{J}$ Clin Oncol 5(3): 283-298, 2014.

35 Koo MM, von Wagner C, Abel GA, McPhail S, Rubin GP and Lyratzopoulos G: Typical and atypical presenting symptoms of breast cancer and their associations with diagnostic intervals: Evidence from a national audit of cancer diagnosis. Cancer Epidemiol 48: 140-146, 2017.
Received October 5, 2018

Revised October 29, 2018

Accepted October 31, 2018 